\title{
TTR
}

Traduction, terminologie, re?daction

\section{Lecture finie du texte infini : Galaxies de Haroldo de Campos}

\section{Inês Oseki-Dépré}

Volume 12, numéro 1, 1er semestre 1999

Poésie, cognition, traduction I

Poetry, Cognition, Translation I

URI : https://id.erudit.org/iderudit/037356ar

DOI : https://doi.org/10.7202/037356ar

Aller au sommaire du numéro

Éditeur(s)

Association canadienne de traductologie

ISSN

0835-8443 (imprimé)

1708-2188 (numérique)

Découvrir la revue

Citer cet article

Oseki-Dépré, I. (1999). Lecture finie du texte infini : Galaxies de Haroldo de Campos. TTR, 12(1), 131-154. https://doi.org/10.7202/037356ar

\section{Résumé de l'article}

Lecture finie d'un texte infini : Galaxies de Haroldo de Campos - Cet article porte sur le fragment 45 des Galaxies de Haroldo de Campos dans sa traduction française considérée comme texte original. Dans la première partie, l'auteure interroge l'aspect météorique du texte afin de démontrer de quelle façon le poème est à la fois mémoire et devenir de la langue. Dans un deuxième temps, la question de l'intertextualité latente dans le fragment est abordée comme savoir sur la littérature. Il s'agit de montrer, à travers une analyse de l'aporie de la traduction, que si le texte porte un savoir sur la littérature, il comporte d'autres éléments cognitifs à décoder lors de sa réception. L'écriture haroldéenne est ainsi appréhendée dans sa réappropriation des structures baroques.
Tous droits réservés @ C TTR: traduction, terminologie, rédaction — Les auteurs, 1999
Ce document est protége par la loi sur le droit d'auteur. L'utilisation des services d'Érudit (y compris la reproduction) est assujettie à sa politique d'utilisation que vous pouvez consulter en ligne.

https://apropos.erudit.org/fr/usagers/politique-dutilisation/ 


\section{Lecture finie du texte infini : Galaxies de Haroldo de Campos}

\section{Inês Oseki-Dépré}

\section{Introduction}

L'exposé qui suit porte sur un fragment des Galaxies $\left(n^{\circ} 45\right)$ de Haroldo de Campos dans sa traduction française considérée comme texte original'.

Nous passerons vite sur la première partie qui vise à montrer que l'aspect météorique du texte n'est là que pour mieux dissimuler son organisation syntaxique et poétique, condition préalable à toute traduction ${ }^{2}$. L'analyse sera rapide pour avoir été effectuée déjà sur la version française et les éléments communs transposés de langue à langue. Nous examinerons les éléments spécifiques plus loin.

Dans un deuxième temps, sera abordée la question de l'intertextualité - latente dans le fragment - comme savoir sur la littérature, qui est le point qui nous intéresse ici. Cette intertextualité se rapporte au passé et à l'avenir, conjuguant la tradition homérique, par la traduction et la parodie, à la poésie en devenir.

Il s'agit de montrer, enfin, que si le texte porte un savoir sur la littérature, il comporte d'autres éléments cognitifs à décoder lors de sa réception. Cette partie de l'analyse prend en compte les deux langues et montre l'aporie de la traduction (Derrida) : en effet, en dépit de son impossibilité première, (" la traduction est impossible »), la traduction s'avère plus que nécessaire, tout en transformant son auteur et son lecteur (et son traducteur). Pour conclure, nous pourrons poser la question de savoir dans quelle mesure l'écriture haroldéenne est baroque. Ne pourrait-on pas y voir, par ailleurs, une réactivation de l'épopée en tant qu'origine de la poésie ?

\footnotetext{
'À ce sujet, voir W. Benjamin, Henri Meschonnic et al.

2 On pourrait en effet interpréter ainsi les paroles de Léon Robel : le sens du texte, ce qui le rend traduisible étant en fait son organisation (sa structure).
} 


\section{Le texte original}

mais uma vez junto ao mar polifluxbórboro polivozbárbaro polúphloisbos polyfizzyboisterous weitaufrauschend fluctissonante esse mar esse mar esse mar esse martexto por quem os signos dobram marujando num estuário de papel num mortuário num monstruário de papel múrmur-rúmorremurmunhante escribalbuciando você converte estes signos-sinos num dobre numa dobra de finados enfim nada de papel estes signos você os ergue contra tuas ruínas ou tuas ruínas contra estes signos balbucilente sololetreando a sóbrio neste eldorido feldorado latinoamargo tua barrouca mortopopéia ibericana na primeira posição do amor ela ergue os joelhos quase êmbolos castanho-lisos e um vagido sussubmisso começa a escorrer como saliva e a mesma castanho-lisa mão retira agora uma lauda datiloscrita da máquina-deescrever quando a saliva já remora na memória o seu ponto saturado de perfume apenas a lembrança de um ter-sido que não foi ou foi não-sendo ou sido é-se pois os signos dobram por este texto que subsume os contextos e os produz como figuras de escrita uma polipalavra contendo todo o rumor do mar uma palavra-búzio que homero soprou e que se deixa transoprar através do sucessivo escarcéu de traduções encadeadas vogais vogando contra o encapelo móvel das consoantes assim também viagem microviagem num livro-deviagens na segunda posição ela está boca-à-terra e um fauno varicoso e senil a empala todocoberto de racimos de uva e revoado por vespas raivecidas que prelibam o mel mascavo minado das regiões escuras dizer que essas palavras convivem no mesmo mar de sargaços da memória é dizer que a linguagem é uma água de barrela uma borra de baixela e que a tela se entretela à tela e tudo se entremela na mesma charada charamela de charonhas carantonhas ou carantelas que trelam e taramelam o pesardelo de um babuíno bêbedo e seus palradisos pastificiosos terrorescendo os festins floriletos pois a linguagem e lavagem é resíduo de drenagem é ressaca e é cloaca e nessa noite nócua é que está sua mensagem nesse publiexposto putriexposto palincesto de todos os passíveis excessos de linguagem abcesso obsesso e houve também a estória daquele alemão que queria aprender o francês por um método rápido assimil de sua invenção e que aprendia uma palavra por dia un mot par jour zept mots chaque zemaine $e$ ao cabo de um mês e ao fim de seis meses e ao fim e ao cabo de um ano tinha já tudo sabido trezentas e sessenta e cinco palavras sabidas tout reglé en ordre bien classé là voui là dans mon cul la kulturra aveva raggione quello tedesco e a civilizacão quero que se danem e é sarro e barro e escarro e amaro isto que fermenta no mais profundo fundo do pélago-linguagem onde o livro faz-se pois não se trata aqui de um livro-rosa para almicândidas e demidonzelas ohfélias nem de um best-seller fimfeliz para amadores de amordorflor mas sim de um nigrolivro um pesteseller um horrídeodigesto de leitura apfelstúrdia para vagamundos e gatopingados e sesquipedantes e sestraiunáticos abstractores enfim quintessentes do elixir caximônico em cartapáceos galáticos na terceira posicão ela é signo e sino e por quem dobra 


\section{La traduction de la galaxie 45}

1 une fois de plus vers la mer polifluxborborante polivocobarbare polúphloisbos polyfizzyboisterous weitaufrauschend fluctissonante vers la mer vers la mer mertexte pour qui consonnent les glas cette mer matelottante dans son extuaire de papier mortuairemonstruaire de papier murmur-rumeur-ruante 5 toi scribalbutiant tu convertis ces signes qui sonnent en chant en plein chant de jourdesmorts funiveresse du papier ces signes tu les dresses contre tes ruines ou tes ruines contre ces signes-sons balbusilencieux sololettriste syllabant dans ton eldouloureux fieldorado latinoamer ta barauque mortépopée ibericaña dans la première position de l'amour elle dresse les genoux quasi-gonds 10 d'une flexion châtain-lisse et son vagissement soussoumis s'écoule en salive et la même châtain-lisse main retire maintenant un texte dactyloécrit de la machineà-écrire quand la salive déjà retrace sa trace jusqu'à son point de saturation de parfum à peine le souvenir d'un avoir-été qui ne fut pas ou fut sans être car les glas consonnent pour ce texte qui subsume les contextes 15 et les produit comme figures d'écriture une polyparole contenant toute la rumeur de la mer une parole-buccin qu'homère souffla et qui se laisse transsouffler à travers le successif tintamarre de traductions enchaînées voyelles vogantes contre le jaillissement mobile des consonnes ainsi aussi voyage revoyage le long d'un livre-de-voyages dans la deuxième position elle est bouche-à-terre et un 20 faune variqueux et sénile l'empale tout couvert de grappes de raisin et envoilé de guêpes enragées qui avantgoûtent le miel adultéré miné des régions obscures dire que ces mots cohabitent dans la même mer des sargasses de la mémoire c'est dire que le langage est une eau de lessive une lie de vaisselle et que la toile s'entretoile à la toile et tout s'entremêle dans la même 25 charade chalumeuse des trognes des charognes qui trichent et trébuchent le cauchemasardeux d'un babouin ivrogne et ses parladits pâteux terrorescents les festins-florilèges car le langage est lavage et résidu de drainage est ressac et est cloaque et dans cette nuit nuisible réside son message dans ce publiexposé putriexposé palimpseste de tous les passibles 30 excès de langage abcès obsexionné et il y eut aussi l'histoire de cet allemand qui voulait apprendre la français par une méthode rapide assimil de son invention et qui apprenait une parole par jour un mot par jour zept mots chaque zemaine et au bout d'un mois et au terme de six mois et à la fin et au bout d'un an avait déjà tout appris trois cent soixante cinq 35 mots sus tout réglé en ordre bien classé là oui là dans mon cul la kulturra aveva raggione quelle tedesco et la civilisation je veux qu'elles se fassent foutre et est boue et glu et déchet et amer ce qui bouillonne dans le fondprofond du pélagos-langage où le livre se livre car il ne s'agit pas ici d'un livre-rose pour d'âmes-candides et demipucelles orphélyriques ni d'40 un best-seller finheureuse pour amateurs d'amourdouleurfleur mais bien d'un nigrolivre un pesteseller un horreader-digest de lecture apfelstourdissante pour vagamondes et loups-garous et sesquipédants et sestralunatiques abstracteurs enfin quintessents de l'elixir de la sacrécaboche en mille feuillets galactiques dans la troisième position elle est signe et saigne 50 elle est lasse et glisse et les glas résonnent ${ }^{3}$

${ }^{3}$ Haroldo de Campos, 1970, traduit par Inês Oseki-Dépré, publié par Donguy, Dachy, Luna Park, lecture au Festival de Cogolin, 1985. 


\section{La lecture}

Lors de la publication intégrale des Galaxies, en 1984, Haroldo de Campos en a fait la présentation suivante : " le formant initial des Galaxies (début/fin) est de 1963; le terminal, de 1976 ». Il s'agit d'un " texte imaginé à l'extrême des limites de la poésie et de la prose, pulsion bioscripturelle en expansion galactique entre ces deux formants échangeables et changeants ». Son " aimant thématique est le voyage comme livre ou le livre comme voyage, entendu aussi comme "livre d'essai" ".

Pour une analyse du texte choisi, le fragment 45 , deux considérations s'imposent, la présentation du texte, assez homogène, avec un nombre de lignes équivalent à ceux des autres fragments, entre 45 et 50 lignes, sur une page, l'absence de ponctuation et de majuscules. La deuxième, compte tenu du fait que nous l'avons traduit, c'est la présence à la fois d'un principe d'organisation - qui le place d'emblée dans le domaine du Texte en tant qu'objet sémiotique - et d'un principe de déconstruction - qui le place, comme le veut Haroldo de Campos lui-même, dans le domaine du Texte post-utopique, dont nous donnerons la définition plus loin.

Première partie : Le texte, mémoire et code de la langue.

Dans une première partie nous essaierons de montrer rapidement dans quelle mesure le texte code, selon les termes de Jacques Roubaud " le fait que toute langue est construite à l'aide d'éléments sonores sans signifié dont la concaténation fait apparaître, dans la phrase, le sens » et en quoi le fragment présenté est et n'est pas un poème en vers libres. En d'autres termes, en quoi ce poème est à la fois mémoire et devenir de la langue. Pour ce faire, nous nous aiderons de la théorie de Jakobson ${ }^{5}$.

Jakobson donne du texte littéraire et en particulier du texte poétique une définition complexe que l'on peut résumer selon les trois points suivants :

(a) le texte poétique se caractérise par le «travail » sur la matière signifiante;

(b) par la récurrence sur l'axe syntagmatique des équivalences paradigmatiques (équivalences forme/sens de type similaire ou dissimilaire);

4 "Quelques thèses sur la poétique », Change 6, Seuil, Paris, 1970, p. 19.

${ }^{5} \mathrm{La}$ 《 théorie » de Jakobson se déduit de sa définition de la fonction poétique Essais de Linguistique Générale, Gallimard, Paris, 1970, et de ses nombreuses analyses du texte littéraire. 
(c) par le surcodage parallélistique, soit, la surdétermination des structures récurrentes sur les divers niveaux où elles se réalisent.

Est par conséquent poétique le texte qui remplit ces trois conditions et c'est dans ce sens qu'il se prête à l'analyse sémiotique dont l'objet, la litterarité, constitue l'invariante qui se trouve dans toute littérature, qui se trouve ainsi formellement définie.

Sans nier la validité de cette théorie, qui est la première à permettre d'effectuer le saut qualitatif dans le domaine de la théorie littéraire, la question qui se pose aujourd'hui au sémioticien va plus loin et concerne le problème de l'écriture, en d'autres termes, le problème de la relation du sujet-écrivain (non plus l'Auteur doué de pouvoirs extraordinaires de la vision romantique et post-romantique) au Texte, et également, celle du Texte au contexte, au monde.

L'analyse-lecture que nous nous proposons de réaliser sur le texte de Haroldo de Campos, vise ces deux aspects de la question.

\section{$\underline{\text { 1. Analyse du fragment }}$}

La première remarque à faire sur le fragment que nous analyserons concerne sa non-conformité aux règles formelles du texte poétique traditionnel. Ainsi, il est impossible de le classer à priori parmi les formes connues.

Le fait que le texte ne possède pas de forme fixe ne nous empêche pas - dans la mesure où nous le considérerons comme un objet sémiotique - de tracer les lignes provisoires qui délimitent ses unités. Pour cela nous nous servirons du critère syntaxique et ce bien que l'unité-ligne, qui découle de la distribution intentionnelle des " vers » sur la page soit soumise à un règlement rythmique ${ }^{6}$. Les unités ainsi dégagées permettent, par ailleurs, de considérer à la fois le texte original et sa traduction, dans la mesure où c'est par le lexique et la prosodie que l'on retrouve leurs éléments différentiels, analysés plus loin. De plus, si la ligne est l'unité formelle du poème, c'est la phrase qui permet de le traduire.

Nous ne manquerons pas de proposer plus loin, il va sans dire, une vision synthétique de l'ensemble. Nous pouvons ajouter aussi que si notre lecture/analyse prend la syntaxe ${ }^{7}$ comme point de départ, elle

${ }^{6}$ « Le seul trait formel qui caractérise le vers libre comme type de poésie est $l e$ fait d'aller à la ligne ", J.R., loc.cit., ibidem.

${ }^{7}$ La structure syntaxique est par ailleurs l'élément commun aux deux langues d'une part en raison de leur origine latine commune, d'autre part, en raison du caractère très elliptique de la syntaxe du poème. "Le poème, ici, est phrase ", 
n'exclut pas, au contraire, les autres paramètres qui constitueront la littérarité du Texte choisi (phono-sémantique).

Notre deuxième remarque a pour objet de préciser la façon dont nous nous y prendrons pour effectuer telle analyse. Celle-ci se limite, pour les besoins du présent exposé, à la première phrase complexe du Texte, prise comme "échantillon ${ }^{8}$. Elle se veut, par ailleurs, très simple et peut être tenue pour une analyse de type traditionnel, ayant recours par moments à quelques apports linguistiques proposés par Nicolas Ruwet dans ses analyses.

\section{P.C.I :}

Principale : « uma vez mais junto ao mar...você converte estes signos-sinos num dobre " (" une fois de plus ... tu convertis ces signes qui sonnent en chant... ») (1.1-1.5)

Coordonnée : "você os ergue contra tus ruinas... " (" tu les dresses contre tes ruines... » (1.6-1.8)

Nos deux propositions (Principale et Coordonnée), bien que quantitativement distinctes (5 lignes et 3 lignes), peuvent être considérées comme parallèles car elles présentent en commun : le même sujet "tu ", le même complément "signes" (" ces signes", "les $»)$ et le verbe au même temps (présent de l'indicatif), tous deux transitifs (" convertis », " dresses ").

Des deux propositions, la seconde peut être considérée comme une expansion de la première, a partir de la transformation «ces signes » — " les ", et on peut les représenter par le schéma :

$$
\mathrm{Pl}=\text { exposition }-\mathrm{P} 2=\text { expansion. }
$$

Ajoutons que le complément de la principale «signes " (qui sonnent) est en rapport avec la proposition antérieure intercalée « pour qui consonnent les glas » ( sonnent $» / \ll$ consonnent $»)^{9}$. Par ailleurs, l'expansion peut être dédoublée en deux propositions (unies par la conjonction " ou »), dont la seconde se caractérise par la présence d'un noyau implicite (*tu dresses tes ruines contre tes signes).

Une fois dégagée la structure syntaxique de cette première phrase complexe (P.C.I.) qui constitue, à notre avis, la première partie du texte (la matrice), essayons de voir dans quelle mesure

J.R., loc.cit., p. 20.

${ }^{8}$ L'analyse de l'échantillon peut évidemment se poursuivre.

9 En portugais « dobram », « sinos » (traduit en français par un verbe). 
l'organisation du noyau est surdéterminée par des sous-organisations prosodiques ou phoniques du texte.
A B
C
D E
F $\quad G \quad H$
I J
K

escribalbuciando você converte estes signos-sinos num dobre numa dobra de finados você ergue estes signos/os contra tuas ruinas

tuas ruinas contra esses signos

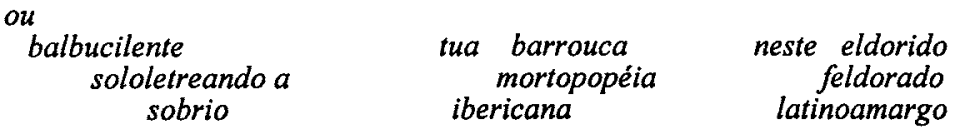

toi scribalbutiant tu convertis ces signes qui sonnent en chant

en plein chant des

jourdemorts

du papier

funivresse

jeudemots

tu dresses ces signes

contre tes ruines

ou

balbusilencieux

sololettriste

syllabant tes ruines contre ces

signes-sons

D'emblée nous notons qu'au parallélisme syntaxique correspond un parallélisme prosodique, quoique asymétrique, surtout en français. La prosodie est marquée ici par les récurrences rythmiques et sonores. En effet, à la première forme gérondive "scribalbutiant » (escribalbuciando) s'oppose une forme transitive "syllabant ta..." (sololetreando $a$ ) qui se trouve, de plus, renforcée par le syntagme «balbusilencieux sololettriste» (balbucilente, avec déplacement du verbe néologique vers l'adjectif) déterminant, et allongée par le 
circonstant de lieu "dans ton eldouloureux fieldorado latinoamer » (neste eldorido feldorado latinoamargo).

Les deux formes gérondives dont il est question (C) sont constituées de mots-valises qui contiennent la même substance sémique :

scrib+ (b) albutiant

syllab+ ant (balbu+silencieux/solo+lettriste), soit, l'acte d'écrire et de lire de façon appliquée (+ difficulté).

Nous avons déjà évoqué les classes (D) et (E) qui n'offrent pas de particularités. La classe (F) offre un faux parallélisme double : " ces », " ces »/« tes », " ta » - mais le singulier du second possessif indique une opposition au pluriel de la première forme. En réalité, " ces » et « tes » se trouvent en distribution complémentaire et «ta " introduit une asymétrie, étant le déterminant du complément de " syllabant » et non pas de " dresses ". Par ailleurs, " ces » est toujours suivi de " signes » et «tes" de « ruines », tandis que « ta " précède " morépopée ».

Les trois unités de (G) sont également des mots-valises : ba+rauque/morto+(é)popée/iberica(na).

C'est dans la classe (G) également que se trouvent les compléments de $(\mathrm{E})$, mais tandis que dans sa première occurrence " signes " (c.o.d.) se trouve déterminé par une relative " qui sonnent ", dans le second cas, il vient antéposé au verbe et est repris par le pronom " les ». Par ailleurs, la classe (G) est en rapport avec la classe (I) dans le jeu permutatoire entre " signes » $X$ « ruines » / « ruines » $X$ " signessons ".

À ce propos, nous pouvons remarquer encore la similitude (phono-sémantique) entre :

$\begin{array}{lll}\begin{array}{l}\text { " eldouloureux } \\ \text { " barauque }\end{array} & \text { fieldorado } & \text { latinoamer " (G) } \\ \text { ibéricana " (I) }\end{array}$

qui se trouvent dans un rapport d'équivalence syntagmatique, les deux classes étant précédées du possessif singulier (《ta »/ «ton »(J)).

\section{Scansion de l'ensemble}

Nous proposons ici notre scansion du texte en ses parties constitutives en vue de l'harmonisation des divers niveaux qui se retrouvent dans le texte littéraire. 
Il n'est pas inutile d'insister sur le fait que notre division, apparemment arbitraire - puisque rien n'empêcherait de considérer le texte comme une longue phrase unique ${ }^{10}$ - se justifie pour des raisons méthodologiques, dans la mesure où elle possède l'avantage de souligner la présence dans le texte d'unités poétiques récurrentes à tous les niveaux. complexes :

Le texte peut ainsi être divisé en quatre "phrases"

I(a) « uma vez mais junto ao mar... você converte estes signos... papel » (1.1 à 1.6) « une fois de plus vers la mer...tu convertis ces signes... du papier » (1.l à l.6)

l(b) " estes signos você os ergue... ibericana » (1.6 à 1.9) " ces signes tu les dresses... ibéricana » (1.6 à l.9)

2(a) " na primeira posição do amor... ou foi não sendo » (1.9 à 1.13) " dans la première position de l'amour... ou fut-sans-être " (1.9 à l.15)

2(b) "pois os signos dobram... num livro de viagens " (l.15 à l.19) " car les glas consonnent... le long d'un livre-de-voyages » (1.15 à 1.20)

3(a) " na segunda posição... regiões escuras » (1.20 à 1.22) " dans la deuxième position... des régions obscures » (1.20 à 1.23$)$

3(b) " dizer que essas palovras... os festins floriletos " (1.20 à 1. 27) " dire que ces mots coalisent... les festins florilèges » (1.23 à 1.29)

3(c) "pois a linguagem é lavagem... abcesso obsesso » (1. 27 à 1.30) " car le langage est lavage... abcès obsexionné " (1.29 à l.32)

3(d) «e houve também... quello tedesco » (1.30 à 1.36) " et il y eut aussi l'histoire... ce tudesque-là » (1.32 à 1.38)

3(f) " pois não se trata... cartapáceos galáticos " (1.36 à 1.43) « car il ne s'agit pas ici... en mille-feuillets galactiques » (1.40 à l.46)

4 " na terceira posição ela... e por quem dobra» (1.44) " dans la troisième position... et les glas résonnent " (1.46 à 1.47).

Bien que le critère qui nous permet de diviser le texte en 4 phrases complexes soit, comme nous l'avons dit, le critère syntaxique (classique), du point de vue sémantique, toutefois, nous aurions intérêt à considérer les 5 parties suivantes :

P.C.I. : Introït (matrice)

${ }^{10} \mathrm{Ce}$ que confirmerait, si nécessaire, l'absence de ponctuation... 
P.C.II : premier mouvement

P.C.III : deuxième mouvement $(\mathrm{a}, \mathrm{b}, \mathrm{c})$

Parenthèse ou troisième mouvement $(\mathrm{d}, \mathrm{e}, \mathrm{f})$

P.C.IV : Finale ou quatrième mouvement.

Nous avons affaire donc à des structures superposantes.

Car si du point de vue syntaxique, chaque partie se caractérise par la présence initiale d'un circonstant de lieu :

" une fois de plus vers la mer »-I

" dans la première position "-II

" dans la deuxième position " -III

" dans la troisième position " -IV,

la Parenthèse, toutefois, nous semble avoir une fonction sémantique distincte (inclusion-exclusion de l'ensemble) sans posséder cette marque formelle.

À l'opposé, l'Introït s'oppose à l'ensemble du texte par l'absence de continuation explicative introduite par "car », que l'on trouve dans la Parenthèse.

" car les glas consonnent "-II

« car le langage est lavage »- III

" car il ne s'agit pas ici d'un livre-rose »-III.

Les niveaux syntaxique et sémantique présentent ainsi quelques décalages significatifs de la structure en contrepoint de l'ensemble du Texte. Le IV mouvement (Finale), interrompu, ne contient pas d'explicative explicite, tandis que le $\mathrm{II}^{\mathrm{e}}$ (P.C.III) en contient deux. Comme nous le montrerons par la suite, le motif de ces propositions est, d'une manière de plus en plus métalinguistique, le livre, le langage et les signes.

$\mathrm{Du}$ point de vue syntaxique également, nous pouvons remarquer que les trois propositions ayant "elle " comme sujet se comportent de façon analogue : circonstant de lieu + Noyau + coordonnée (" et ") + coordonnée (" et ») :

elle + verbe (présent de l'indicatif).

" elle dresse les genoux 》 + " et son vagissement... s'écoule »

" et la... main retire... un texte "

(ela ergue os joelhos $+e$ um vagido... começa a escorrer)

(e a... mão retira... uma lauda)

" elle est bouche-à-terre + " et un faune... l'empale "

(ela está boca à terra + e um fauno... a empala)

" et survolé... » 
" elle est signe + et saigne »

(ela é signo + e sino)"

" elle est lasse + et glisse $»^{12}$ (e revoado...")

+ "et les glas résonnent »

$(+e$ os sinos dobram")

La dernière proposition (" elle »), qui ne contient pas de suite explicative, offre "en compensation 》 un exemple de dédoublement parallélistique d'autant plus riche qu'il se réalise au moyen d'équivalences phono-prosodiques (attribut/prédicat) avec une configuration chiasmatique au niveau des voyelles (signe/saigne//lasse/glisse).

Du point de vue lexical, nous pouvons remarquer également la transformation du verbe d'action (" dresse ") en verbe d'état (" est »), jusqu'à l'évanouissement du sujet « elle ».

Dans les trois cas, les coordonnées n'ont pas « elle " comme sujet et on passe de la métonymie (" vagissement ", vagido) à l'Autre, agent extérieur, d'abord métonymique ("la main ", a mão), mythologique ensuite (" un faune ", um fauno), métalinguistique enfin ("les glas", os sinos). De même, les propositions relatives ne se rapportent jamais à « elle » en tant qu'antécédent.

C'est dans la deuxième occurrence de " elle " (P.C.III) que nous trouvons le plus grand nombre de propositions, distribuées sur deux topiques, que nous pourrions appeler " intérieur " et " extérieur ». En effet, nous $y$ trouvons respectivement une réflexion métalinguistique :

que),

"dire que ces mots... c'est dire... et que » (dizer que... dizer avec une structure verbale " infinitive » et une parenthèse anecdotique, insertion du récit dans le discours, avec son système achevé du point de vue du sujet, des temps verbaux et des déictiques, introduite de façon impersonnelle :

" et il y eut aussi l'histoire... » ( $e$ houve também a história...)

1 En portugais : elle est signe et glas, remplacé ici par saigne par « contamination sémantique » (glissement du signifié).

${ }^{12}$ La structure syntaxique est identique en portugais : pois, pois, pois (car), ela (elle). Ce dernier vers est ajouté pour le rythme. 
Il serait quasi superflu de noter la surdétermination de ce découpage par la structuration du niveau prosodique (rythme, sonorités). A la régularité syllabique sous-jacente correspond en effet une grande quantité d'assonances, allitérations, voire des paronomases, en portugais sur les voyelles /i/ (lisos, vagido, sussubmisso, saliva, lisa, retira, datiloscrita, sido, sido, signos, escrita) et /e//E/ (ela, ergue, joelhos, êmbolos, começa, escorrer, escrever), en français par les consonnes /s/ (dresse, flexion, lisse, vagissement, soussoumis, s'écoule, salive, lisse, texte, salive, retrace, trace, saturation, souvenir, sans, consonnent, subsume, contextes) et $/ R /$ (dresse, retire, dactyloécrit, écrire, retrace, trace, parfum, produit, écriture, polyparole, etc. $)^{13}$.

\section{La Parenthèse}

Dans le passage fonctionnellement distinct que nous avons appelé Parenthèse, les parallélismes se présentent non pas sous forme de succession syntagmatique mais plus nettement sous forme de paradigme :
" une parole par jour » (um palavra por dia)
" un mot par jour »
" sept mots chaque semaine"
" et au bout d'un mois " (e ao cabo de um mês)
" et au terme de six mois " (e ao fim de seis meses)
"et à la fin et au bout d'un an ». (e ao fim e ao cabo de um ano)

Le parallélisme syntaxique se présente sous la forme de relatives également :

" qui voulait»// et qui apprenait»/(que queria le que aprendia)

Nous pouvons ajouter à ces exemples les rimes imparfaites (assonances) en français :

«boue »/« glu» (en portugais : sarro e barro e escarro e amaro)

" déchet »/« amer»"

" fondprofond 》 et « le livre se livre » (profundo fundo/ o livro faz-se). composés :

Les récurrences se présentent sous la forme de mots

${ }^{13}$ Selon le principe de " compensation ». 
« livre-rose »/« demipucelles candides $» / «$ best-seller $»$ orphélyriques »/« âmes« finheureuse $) / \ll$ nigrolivre $» ~ «$ pesteseller $» / \ll$ horreader-digeste $»$ et/ou des motsvalises: "vagamondes 》, " sesquipédants ", " apfesltourdissant », " quintessents » " sacrecaboche », etc., dans un procédé d'énumération amplifiante.

L'intérêt de cette scansion syntactico-sémantique réside en ceci qu'elle permet de mieux appréhender les parallélismes formels entre les grandes (et les petites) unités syntaxiques du texte et par conséquent le travail d'encodage (mise-en-code) effectué par le poète qui produit, en les dissimulant, ses unités surdéterminées.

Nous pouvons mettre en évidence ainsi les équivalences qui assurent la cohésion de chaque unité (mouvement) du texte et qui établissent en même temps leur lien et l'unité de l'ensemble. Unité qui ne signifie ici ni linéarité ni fermeture mais ce qui donne la consistance matérielle à ce texte en spirale, dont la dynamique ne se fige que l'espace d'une lecture.

Enfin, cette analyse permet de clarifier à rebours le processus critique opéré par une traduction de type littéral, pris dans le sens moderne du terme ${ }^{14}$. Une traduction où gain et perte s'équilibrent dans la mesure où tous les paramètres du texte sont pris en compte dans le processus traductif.

L'affirmation de Jacques Roubaud, pour qui la littérature est code formel et mémoire sémantique d'une langue, acquiert une nouvelle dimension dans cet espace galactique. Le texte d'Haroldo de Campos code et transforme les données prosodiques et sémiques de la langue (retransformées par notre traduction). Il les transforme, puisqu'il fait exploser les limites d'une langue en intégrant dans les mêmes paradigmes une série de néologismes et/ou de mots-valises d'autres idiomes montrant ainsi que la fusion se fait dans le langage mais profondément (inconsciemment) dans le rythme du langage-discours.

Deuxième partie : Le texte comme théorie du texte et comme scovoir de la littérature.

La deuxième partie de notre analyse-lecture a comme objectif non plus le texte comme objet sémiotique (poétique) en soi, mais son insertion dans le contexte historico-littéraire, en d'autres termes, dans la tradition d'une part, dans l'intertextualité de l'autre. Cette interprétation est, bien sûr, induite par l'analyse précédente ${ }^{15}$, elle en découle.

14 Voir Inês Oseki-Dépré, Théories et Pratiques de la Traduction Littéraire, Armand Colin, coll. U, Paris, 1999, en particulier le chapitre consacré aux théories prospectives. 
Nous examinerons donc en premier lieu la manière selon laquelle le texte original (en nous référant au seul texte traduit) contient sa propre théorie et puis dans quelle mesure il cherche à transformer la littérature et la réalité.

\section{Le métalangage}

Revenons au début du poème, que nous appellerons Introduction ou Introït, à la ligne 1 :

mais uma vez

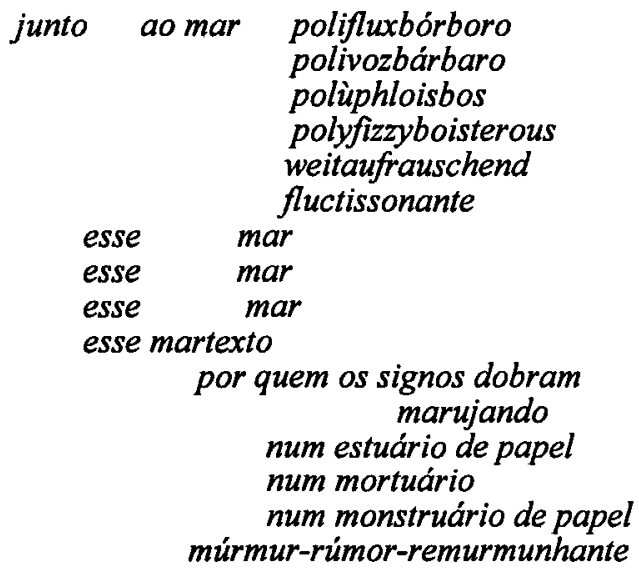

${ }^{15}$ Qui correspond au commentaire de l'opération traduisante et à la vérification des hypothèses avancées... 
dans son estuaire de papier

mortuairemonstruaire de papier

murmur-rumeur-ruante

Ici nombreuses sont les équivalences phono-sémantiques paradigmatiques juxtaposées sur l'axe du syntagme qui soulignent le métalangage. Quelques remarques s'imposent quant au nombre de syllabes assez régulier des déterminants de «mer » $(6,6,5,7,4$ et 4$)$; ainsi qu'au signifié invariant et leur signifiant polyglottique et homophone, dans un fonctionnement pair (similaire).

La même équivalence se trouve dans les récurrences « vers la mer » et dans le circonstant de lieu qui suit " matelottante », constitué par une énumération amplifiante (" estuaire de papier ", "mortuairemonstruaire de papier 》). La séquence " murmur-rumeurruante ", qui fournit la rime à " fluctissonnante " et à " matelottante " suit le chemin inverse - car elle débute par un « demi " mot -, se poursuit ensuite sur un mot plein qui l'allitère (et en quelque sorte l'anagrammatise) et enfin sur le mot " ruante » qui le complète en lui ajoutant un sens actif (" verbal »). La traduction, assez littérale dans l'ensemble, remplace néanmoins "remurmunhante " par " ruante " dans un hommage (clin-d'oeil) non gratuit à la « nixe " mallarméenne. Le motif de la « mer » est, comme nous le verrons par la suite, essentiel (la mer de papier).

\section{Un sujet non-lyrique}

Comme nous l'avons vu au préalable, le texte est adressé à " tu 》 dès l'Introìt. Or, " tu " est le sujet, extrêmement ambigu, de cette partie, pouvant désigner tu-lecteur ou toi-qui-écris (les deux occurrences étant volontairement confondues et réciproquement identifiables). Comme on ne le sait que trop depuis Benveniste, à partir du moment où l'on pose «tu », l'on présuppose la présente instance de "je», sujet de l'énonciation.

" Tu » place ici la question de l'énonciation dans l'acte de la parole-écriture. Le texte se situe ainsi au niveau du discours et non pas de l'énoncé qui, sous la fausse notion de l'objectivité, est ce qui se prétend plus proche de la "vérité », alors que dans la fiction ${ }^{16}$ la seule

16 Haroldo de Campos rappelle Wolfgang Iser qui, dans "Akte des Fingierens ", associe fiction et langage. Pour la tradition de la poésie, cette relation n'a jamais cessé d'être étroite. " Poeta - poetas: es quier dezir tanto como fallador de nuevo de razón, e enfennidor dela... ". Cette définition du métier du poète dans la General Estoria du roi-troubadour Alfonso, El Sabio (1221-1284), peut être reprise par celui qui veut donner une épaisseur 
vérité consiste dans le tissu discursif qui se laisse surprendre ou qui se montre en s'énonçant, dans le geste même de l'écriture. Ce fait est confirmé par la présence massive des déictiques que nous avons déjà mentionnés ( ces », « tes », « ta »).

Le sujet de l'énonciation demeure présupposé dans l'allocuteur ( tu $»)$, mais surtout dans les formes déictiques de la première personne tout le long du texte. Il devient explicite à la fin (III mouvement), sous la forme " je veux » qui non seulement indique la présence du sujet énonciateur mais la concomitance du dire et de l'action (performatif) : en disant « je veux $», j$ 'agis.

Ainsi, du « je » implicite de « cette mer » (1.3), " toi-tu » (l.et 1.7), « ces (signes) » (1.5 et 1.6), « tes (ruines) » (1.7), " ces (signessons) 》 (1.7), " ton (fieldorado) » (1.8), « ta (mortépopée) 》 (1.9), " maintenant » (1.12), " ce (texte) » (1.30) " ça » (1.38), « ici » (1.40), nous arrivons au « je » explicite de « je veux ». Mais la phrase finale rend le texte à l'anonymat, à la personne absente (" elle ») et exclut, en le conduisant à son terme, l'appropriation du discours par un " je ». On verra plus loin que cette non- appropriation soulignée par un jeu apparemment énonciatif mais plutôt métalinguistique prend la forme d'une citation intertextuelle.

En même temps, le premier mouvement introduit la nonpersonne, " elle ", objet dont on parle dans le texte. Cet embryon de fiction, dont la tonalité sémique est érotique, vient se fondre et s'évanouir (" la même châtain-lisse main ») dans l'acte d'écrire, dans la machine-à-écrire.

La suite, au niveau de la proposition coordonnée introduite par " car » (troisième paradigme), marque une nouvelle scène de l'écriture : le retour du sujet implicite mais surtout l'insistance d'une lucidité métalinguistique et quasi-théorique quant à l'activité productrice de la pratique signifiante : "car les glas consonnent pour ce texte qui subsume les contextes et les produit comme figures d'écriture... »

Le deuxième mouvement ré-introduit « elle », qui réapparaîtra dans le Finale, toujours dans le même contexte :

« dans la... position » (première, deuxième, troisième...)

" Elle » - mystérieuse - victime d'une mise-en-scène/miseen-signe, passive et agressée et qui se transforme en signes par la main (châtain-lisse) du poète. « Elle », non pas l'actrice, mais peut-être une

diachronique aux idées si typiquement propres à la tranchante coupure de la modernité comme celle de l'histrion littéraire de Poe et celle du poète simulateur de Pessoa ("fingidor») en passant par le couple fiction et fictif: " un tour ou jonglerie (tout Art en est là)... — - dans la poétique de Mallarmé. 
actrice dans le script de la mémoire, elle représente le fil ténu de la fiction, créée, recréée et consumée dans le discours, selon le mot de Thompson : "Whatever I am talking about, I am also talking about literature ". Dans le texte, la référence au discours littéraire est à chaque fois explicitée :

" un texte dactyloécrit...trace » (I)

" dire que ces mots... " (II)

« elle est signe... » (III).

Le troisième paradigme (présent dans les trois mouvements) présente la particularité, déjà signalée, d'éclaircir le texte, de le situer à un niveau davantage théorique. En effet, nous pouvons « traduire » les trois propositions :

I- car les glas consonnent

II- car le langage est lavage

III- car il ne s'agit pas ici d'un livre-rose

de la manière suivante : les signes, convertis par "toi-je » en glas consonnent; elle, signe, converti par "toi-je», consonne, mais les signes-glas consonnent pour les signes-sons convertis par «toi-je ». L'aspect funèbre du vers de Donne disparait ici, certes, au profit de la paronomase " consonner " qui n'a pas cette connotation en français.

En d'autres termes, le langage est la mémoire, et justement pour cela, il est le lieu de coexistence de boue, de lie, de cloaque, mais dans le langage, le travail sur la matière signifiante est le seul acte à pouvoir "laver " le langage, en faire évacuer la "civilisation" et effacer les mots inutiles en en créant d'autres et en retenant les traces de la mer d'Homère, des voyelles et des consonnes.

La dernière proposition définit le livre - car les Galaxies sont le livre : un nigrolivre, un pesteseller, un horreaderdigest, le livre qui corrode et récuse la fin heureuse mais qui récuse la fin récuse le début et toute la conception de la littérature traditionnelle, logocentrique, ainsi que la fable, l'Auteur, les ingrédients habituels du répertoire canonique. Cette dernière proposition est mise en relief par sa structure négative (" il ne s'agit pas ici") suivie d'une double affirmation (" mais bien »). Et si, comme le propose J. Roubaud, la poésie en vers libre annonce le Cercle linguistique de Prague et les travaux formalistes, la poésie post-utopique d'Haroldo de Campos s'avance dans le sens de la dissémination derridéenne.

Il nous reste, dans cette partie, à commenter l'unité parenthétique narrative, la seule à présenter entièrement les apparences formelles de la fiction.

Nous avons déjà mentionné la relation entre les unités : 
dans la... position

/ $\quad$ car...

1

II

où I et II se retrouvent dans une conception théorique de la littérature (post-utopique) $)^{17}$.

Or, ici le sujet tout en étant la troisième personne (" l'histoire de l'allemand ") comme en I et en II, s'oppose aux autres en tant que négativement marqué du point de vue sémantique. Il s'agit d'un "personnage » sot, le personnage de l'anecdote, car il représente le consommateur (le capitaliste), la langue (et la conception de la langue comme une nomenclature cumulable, comme un dictionnaire poussiéreux) : tant de mots par mois, par an, dans le but de s'approprier un idiome autre. L'attitude ainsi critiquée est l'antithèse de la théorie du Texte, lequel fait parler d'autres langues dans sa langue.

C'est ce qui explique que la relation entre l'anecdote formellement distincte du restant du texte - et la continuation se présente sous la forme d'une rupture : "je veux qu'elles se fassent foutre" (en portugais, quero que se danem).

Au total, le texte, caractérisé par la récurrence, le maintien et la transformation cyclique de ses unités, offre une théorie de la pratique littéraire (écriture et traduction) étayée par le jeu des personnes qui y fonctionnent comme fil conducteur.

Le sujet présupposé, le sujet énonçant, s'occulte mais révèle son occultation d'être investi par la dialectique ludique (aporétique?) des divers pronoms dans la distribution suivante :

THÉORIE

PRATIQUE

FICTION $^{18}$

toi

cette

elle

17 Terme qu'Haroldo de Campos préfère à " post-moderne» en ceci qu'il correspond à un moment de l'Histoire. Ce moment est celui de la perte de l'Espérance révolutionnaire qui fonctionnait comme le moteur idéologique d'une révolution formelle. Le post-utopique n'est pas le rejet de l'action mais la proposition d'une action différente. Voir "Pos-tudo », poème d'Augusto de Campos, et Haroldo de Campos, "Poesie e modernidade », dans $O$ arco-iris branco, Imago, Rio, 1998.

18 « Théorie» renvoie aux éléments métalinguistiques de l'énonciation; " pratique » à la présence du sujet et « fiction », aux éléments de la fable. 


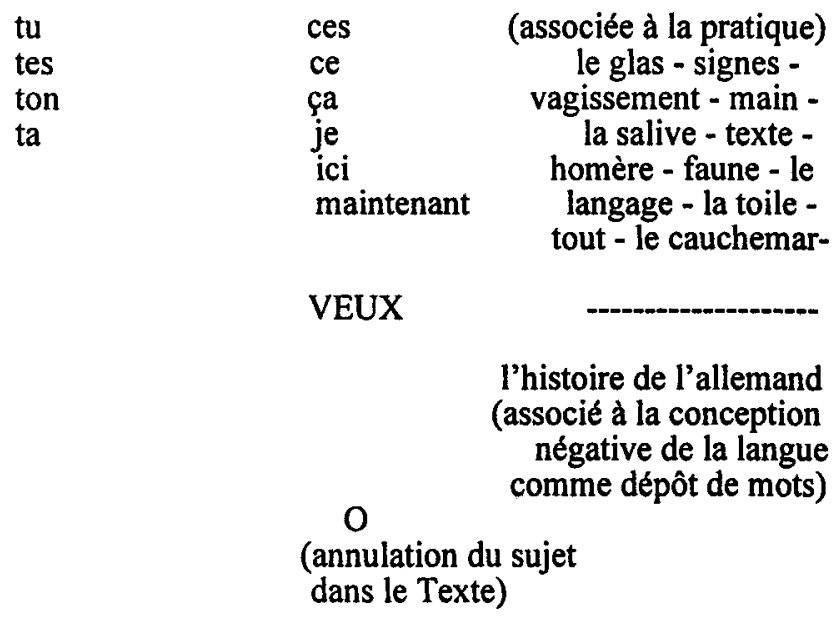

\section{Tradition et intertextualité}

(a) L'itinéraire parcouru par le sujet, qui englobe (tel Lautréamont) le lecteur dans sa pratique à travers le pélagos-langage, mer où la vieille culture du capitalisme linguistique (par l'intermédiaire de l'étranger) se noie dans la production signifiante, fait apparaître une présence féminine aussi fictive que diffuse et, ce qu'il en reste, c'est le texte dans son énonciation, par laquelle un vouloir (un désir?) s'affirme, en un éclair.

Le texte ainsi participe des traces qui, pour être contemporaines, ne renient pas pour autant leur histoire. Traces d'une écriture qui engendre sa propre théorie, théorie nouvelle du langagepensée. La littérature (la poésie) poursuit sur la voie de la transformation des vieilles structures, annulant de ce geste le sujet en tant que conscience suprême (logocentrique), maître de ses personnages et de ses fables.

Cette désappropriation du discours se manifeste également à travers un tissu citationnel, ainsi " pour qui (con-)sonnent les glas "), est la reprise du vers de John Donne ( From whom the bells toll »), luimême ré-utilisé par Hemingway dans son roman et plus tard récupéré par Hollywood (ce qui crée le lien avec la définition du " livre "). De même « ces signes tu les dresses contre tes ruines ou tes ruines contre ces signes-sons 》 est la citation paraphrastique du vers d'Eliot (final du The Waste Land ) : "These fragments I have shored against my ruins 》 à laquelle Ezra Pound répond en contrepoint (Chant VIII) : "These fragments you have shelved (shored) 》. 
(b) En même temps s'esquisse une autre mise-en-scène de l'intertextualité via la traduction. «Poliparole» désigne " polùphloisbos ", Homère, mais la littérature dans son processus historique et son devenir de " traductions voyelles vogantes contre le jaillissement mobile des consonnes ». En outre, ce mot homérien fonctionne comme la pierre-de-touche du texte - Ezra Pound (qui la ré-utilise dans ses poétiques et critiques) -, au moyen du paradigme para-phonique qu'il y induit et également des traductions de Joyce (" polyfizzyboisterous "), de Voss ("weitaufrauschend "), et du brésilien Odorico Mendes (" fluctissonante ") ${ }^{19}$ : toutes des transcréations ${ }^{20}$ poétiques. C'est dire que le texte porte aussi une théorie de la traduction comme moyen (instrument) poétique.

Aussi bien, " poliparole » reprend la série d'épithètes de la mer homérienne et la série " voyelles vogantes", " voyage revoyage le long d'un livre-de-voyages " répond à d'autres textes des Galaxies, comme en un seul souffle.

Les mêmes récurrences se trouvent dans le $\mathrm{II}^{e}$ mouvement, avec configuration chiasmatique, rendues par une tentative de transcréation :

" uma água de barrela uma borra de baixela "

" une eau de lessive une lie de vaisselle ", où l'on trouve la présence des mêmes sonorités inversées.

Les rimes et échos y abondent :

" a tela se entretela à tela »

" la toile s'entretoile à la toile »

"charonhas carantonhas ou carantelas que trelam e taramelam "

" des trognes des charognes qui trichent et trébuchent»,

phrase particulièrement difficile à traduire dans laquelle on pourrait ajouter "des tronches" pour carantonhas; mais où on trouve des néologismes " trelam » (de " trela », dans " dar trela à » : se montrer familier), à côté de mots existants mais rares.

en :

Le même type de configuration prono-prosodique se trouve

19 Odorico Mendes est l'auteur entre autres d'une version de l'Odyssée particulièrement travaillée du point de vue de la prosodie. Haroldo de Campos lui a dédié plusieurs études.

${ }^{20}$ Voir à ce sujet, Haroldo de Campos, « De la traduction comme création et comme critique ", Change, $\mathrm{n}^{\circ} 14$ (Transformer traduire), Seghers/Laffont, février 1973. 
"pois a linguagem é lavagem é resíduo de drenagem " (1.27/28) (" car le langage est lavage est résidu du drainage ")

"é ressaca e cloaca " (1.28) " est ressac et est cloaque " et l'insert que l'on constate en " toile s'entretoile " se répète dans " nuit nuisible ". " Message " et " langage 》 répondent à " car le langage "... mais aussi à " voyage revoyage... ". Il faut noter aussi la configuration allitérante de : " surexcès », " abcès », " obsexionné » (" excessos », " abcessos ", " obsessos »).

\section{(c) Le texte comme questionnement sur la réalité}

Il est difficile d'isoler ce fragment de l'ensemble des Galaxies et il est encore plus difficile d'isoler les Galaxies du projet global d'Haroldo de Campos. Commencé en 63, en pleine période d'Espérance (le groupe Noigandres, 55; Brasilia et le Plan-pilote, 62, année de grande effervescence politique et culturelle au Brésil), l'ouvrage (journal de voyages?) a continué d'être écrit jusqu'en 1976, le fragment analysé datant de 1970, moment de durcissement de la Dictature militaire au Brésil, à tendance néo-fascisante. Une certaine désespérance s'y lit, pour laquelle la consolation reste le poien, le faire (" parce que c'est plus difficile que de ne pas faire », Joấo Cabral de Melo Neto).

On ne peut donc se contenter d'une lecture sémiotique qui confirmerait l'appartenance du texte au domaine poétique, fût-il un hommage à la littérature, imbriqué dans une convocation/dénonciation de la réalité. En réalité, le projet des Galaxies est bien plus vaste, puisqu'il relie l'ancien et le nouveau, mais surtout il propose une nouvelle lecture et de la littérature et de la réalité.

On a parlé d'une écriture baroque à son sujet, ce que nous n'aurions pas de mal à mettre en relief, si on définissait le baroque par la surabondance (concentrée) de mots recherchés, par l'antithèse entre le haut et le bas, et surtout par le souffle. Certes, Haroldo de Campos fait allusion au baroque, mais plutôt comme un regret (comme motif) que par une forme d'écriture et si on voulait interpréter l'expression « ta barauque mortépopée ibericana » en la considérant autant comme " barauque » que " mortépopée ».

Car, pour le poète brésilien, contrairement aux idées reçues sur la culture nationale (qui serait une sous-culture portugaise tentant d'affirmer son nationalisme seulement à partir du XIX ${ }^{\mathrm{e}}$ siècle), le pays est né baroque ${ }^{21}$ : "Je dirai que le baroque pour nous est la nonorigine : nos litteratures, qui ont fait leur apparition avec le baroque, n'ont point eu d'enfance (infans : celui qui ne parle pas). Elles sont nées adultes (comme certains héros mythologiques), parlant un code

21 Voir « De la raison anthropophagique », Lettre internationale, $n^{\circ} 20$ (printemps 1989) et Le séquestre du baroque, Fundação Casa de Jorge Amado, Salvador, Brésil, 1989. 
universel extrêmement élaboré : le code rhétorique baroque (avec des survivances du Moyen Âge tardif et de la Renaissance, décantées, dans le cas brésilien, par le maniérisme de Camoens) 》. Mais cette épopée baroque est désormais morte.

En revanche, nombreuses sont les références à l'épopée, d'abord de façon nommée, "une parole-buccin qu'homère souffla et qui se laisse transsouffler " (uma palavra-búzio que homero soprou e que se deixa transsoprar), puis de façon intertextuelle. Nous avons évoqué les hommages rendus aux poètes de langue anglaise cités dans le fragment, tels John Donne, T.S. Eliot et Ezra Pound. Aussi bien, pourrait-on voir dans le fragment un hommage tacite à Mallarmé et un hommage moins tacite à Fernando Pessoa, poètes qu'Haroldo rapproche par ailleurs dans leur rapport à la fiction. Fernando Pessoa est l'auteur d'un poème, Ulysse, sur le mythe " qui est le rien qui est tout " et dans lequel celui qui "sans exister nous combla et nous créa ». ${ }^{22} \mathrm{Qu}$ 'on pourrait indexer comme l'origine de la littérature.

Pour Ezra Pound, il s'agit de faire comme Homère, d'écrire l'épopée de l'homme moderne en partant d'Homère ${ }^{23}$ et, en parcourant son itinéraire dans le temps (Homère, Properce, Di Cavalcanti, Dante, les troubadours), d'être le "ministre des arts sans portefeuille" (Horace Gregory), d'être une sorte de porte-parole de l'homme occidental (" antenne de la race »).

Or, si Ezra Pound est nommément cité, traducteur (et réanimateur) du passé, notre traduction (en tant que moyen critique, en tant que lecture rapprochée) nous permet de saisir un autre paramètre du texte haroldéen et nous ne pouvons pas ne pas nommer Joyce. Ce qui est intéressant, c'est que Haroldo de Campos ${ }^{24}$ lui-même préconise la méthode " idéogrammique " recommandée par E.P., qui consiste à mesurer le rapport (" influence ») entre deux écritures via la traduction. Ici nous nous trouvons non seulement devant l'allusion à une koiné commune, au voyage d'Ulysse qui chez Joyce est un voyage intérieur, mais à un projet poétique dans tous les sens du mot : les origines du récit, les origines de la poésie, mais aussi les procédés joycéens qui racontent cette origine, les mots barbares, les néologismes, les motsvalises, la citation, la juxtaposition, le dehors et le dedans, l'ironie dans la phrase infinie. En même temps, il s'agit pour le poème de réunir Pound et Joyce, ces deux géants de la poésie qui furent si proches et si lointains ${ }^{25}$.

22 Voir Fernando Pessoa, Mensagem, O.P., Aguilar, Rio, 1965.

${ }^{23}$ Voir les Cantos d'Ezra Pound, qui intègrent les fragments d'Homère.

${ }^{24}$ Voir article cité, p.81.

${ }^{25}$ On sait tout ce que Joyce doit à Pound pour la reconnaissance de son oeuvre, 152 
Selon Forrest Read, « Pound et Joyce sont les deux seuls qui décidèrent dès le début de suivre la vocation classique et de se préparer à écrire une épopée (...) en tant que modernes et en tant que classiques ». "Tous deux développèrent une seule idée en vue d'une forme synthétique toujours plus vaste, plus intégrante » (p.17) et Pound va jusqu'à dire : « Ulysse est ma réponse ».

Ainsi, la référence au baroque ${ }^{26}$ fait partie de cette intention propédeutique : le poème post-utopique est la réécriture d'Homère (via la traduction d'Odorico Mendes; via l'hypertraduction de Joyce, via l'intertraduction de Pound), hommage à la tradition et devenir.

\section{Conclusion}

On pourrait conclure là-dessus aussi bien pour Haroldo de Campos $^{27}$ non sans rappeler certaines considérations qu'il tisse dans sa préface à sa traduction du Finnegans Wake ${ }^{28}$. "Le rythme de cette prosepoésie - riverrun-riocorrente - est quelque chose comme un flux global et continu. Elan-vital. Durchdringung... Une texture où la notion de développement linéaire du récit cesse d'être valide. Tout se passe dans un temps total et dans un espace total. Espacetemps. Le véritable personnage est le langage » (p.23).

Nous pourrions parodier Voltaire, cité par Jacques Delille, traducteur de l'Énéide, autre hypertexte de l'épopée homérique, "si c'est Homère qui a fait Virgile, c'est son plus bel ouvrage ". En effet, si c'est Homère qui a fait Pound, Joyce, Pessoa, Haroldo de Campos, ce

à travers les multiples articles et interventions (y compris de l'aide financière) de ce dernier auprès des maisons d'éditions, revues, personnes importantes (Yeats, Eliot). On sait aussi à quel point Pound voyait dans Joyce un génie sans adhérer tout à fait à son œuvre.

${ }^{26}$ De l'Ulysse de Joyce, Pound dit : " Nous y trouvons Télémaque, son père, les sirènes, le Cyclope, sous des travestissements inattendus, baroques, argotiques, véridiques et gigantesques ", Pound/Joyce, Mercure de France, 1970, p. 228 (C'est nous qui soulignons).

${ }^{27} \mathrm{Ce}$ que confirme un de ses poèmes bien plus récent (1990) où l'identification avec l'Ulysse de Dante est plus claire, l'hybris du poète-voyageur le conduit en enfer. Voir "Finismonde : le dernier voyage", dans Os melhores poemas de Haroldo de Campos, sel. Inês Oseki-Dépré, Global, São Paulo, 1992, traduit par IOD pour le Festival de poésie du Val de Marne, 1995, publié dans Une anthologie immédiate, Fourbis, 1996.

${ }^{28}$ En collaboration avec Augusto de Campos, Panaroma do Finnegans Wake, ed. Perspectiva, São Paulo, 1971. 
sont ses plus beaux ouvrages, à la différence que pour le poète brésilien, Ulysse n'est pas un personnage, mais le poète lui-même ${ }^{29}$.

\title{
UFR LACS - Université de Provence
}

RÉSUMÉ : Lecture finie d'un texte infini : Galaxies de Haroldo de Campos - Cet article porte sur le fragment 45 des Galaxies de Haroldo de Campos dans sa traduction française considérée comme texte original. Dans la première partie, l'auteure interroge l'aspect météorique du texte afin de démontrer de quelle façon le poème est à la fois mémoire et devenir de la langue. Dans un deuxième temps, la question de l'intertextualité latente dans le fragment est abordée comme savoir sur la littérature. Il s'agit de montrer, à travers une analyse de l'aporie de la traduction, que si le texte porte un savoir sur la littérature, il comporte d'autres éléments cognitifs à décoder lors de sa réception. L'écriture haroldéenne est ainsi appréhendée dans sa réappropriation des structures baroques.

\begin{abstract}
A Finite Reading of an Infinite Text : Haroldo de Campos'Galaxies - This article examines fragment 45 of Haroldo de Campos' Galoxies in its French translation considered to be the original text. The author first questions the meteoric side of the text in order to demonstrate how language, in the poem, is at once memory and its state of becoming. The author goes on to question the latent intertextuality in the fragment, seen as knowledge of literature. We see that by way of an analysis of the aporie of translation, that if the text deals with this knowledge of literature, it contains other cognitive elements to be decoded upon the text's reception. Haroldean writing is thus understood in its reappropriation of its baroque structure.
\end{abstract}

Inês Oseki-Dépré : Département de littérature générale et comparée, 5, rue Campra, 13100 Aix-en-Provence, France. Courriel : osekines@aixup.univ-aix.fr

${ }^{29} \mathrm{Ce}$ qui, selon les termes de Pound, l'inclurait dans la catégorie " phallique " donc poétique, - mue par le désir - et non pas "scatologique", donc narratrice, mue par l'analyse et dans laquelle il met Joyce. 